\title{
Synthesis of $N, O$-Type Inherently Chiral Calix[4]arenes Substituted on the Lower Rim and their Organocatalysis Properties
}

\author{
Ming-Liang Chang, Yong He, Jing Zhou* and Shao-Yong Li* \\ Tianjin Key Laboratory on Technologies Enabling Development of Clinical Therapeutics and Diagnostics (Theranostics), \\ School of Pharmacy, Tianjin Medical University, 300070 Tianjin, China
}

\begin{abstract}
This work presented the first study of organocatalytic behaviors of inherently chiral calix[4] arenes substituted at the lower rim. A pair of $\mathrm{N}, \mathrm{O}$-type enantiomers based on inherently chiral calix[4]arenes substituted at the lower rim were readily synthesized and applied to catalyze Henry reaction between aromatic aldehydes and nitromethane. Their organocatalytic reaction can afford the desired products in excellent yields (up to $99 \%$ ) but poor enantioselectivities (up to $7.5 \%$ ee).
\end{abstract}

Keywords: inherently chiral, calix[4]arene, asymmetric catalysis, Henry reaction

\section{Introduction}

Inherently chiral calixarenes as a novel type of chiral macrocyclic compounds have been intensively studied in the past three decades. To mimic enzymatic catalysis process and develop efficient chiral catalysts, a variety of inherently chiral calix[4]arenes substituted at the meta position and the upper rim have been applied as chiral organocatalysts in aldol reaction, ${ }^{1}$ addition of diethylzinc to benzaldehyde, ${ }^{2}$ Michael addition ${ }^{3-7}$ and alkylation of a glycine derivative. ${ }^{7}$ And the highest ee in these catalytic reactions is up to $31 \% .{ }^{6}$ However, until now inherently chiral calix[4]arenes substituted at the lower rim have not been applied in asymmetric organocatalysis although they have been frequently prepared and applied in chiral recognition. ${ }^{8}$ To systematically study the application of inherently chiral calixarenes, it is necessary to explore the organocatalytic behaviors of inherently chiral calix[4] arenes substituted at the lower rim.

Henry reactions are one of the most useful and widely employed methods for carbon-carbon bond formation in organic chemistry. The resulting nitro alcohol (nitroaldol) products can be transformed into a number of nitrogen and oxygen-containing derivatives such as 1,2-amino alcohols, amino sugars, nitro ketones, nitro alkenes, ketones, and other important compounds. ${ }^{9-14}$ It was reported that chiral $\mathrm{N}, \mathrm{O}$-type cupreidine derivatives can perform outstanding catalytical activities for Henry reaction. Mechanistic studies on their catalytic behaviors indicated they serve

*e-mail: zhoujing@tmu.edu.cn; lishaoyong@tmu.edu.cn as acid-base bifunctional organocatalysts through $\mathrm{H}$-bond interactions with the acceptor and donor components of the reactions through the quinuclidine nitrogen and the aromatic hydroxyl, respectively. ${ }^{10,15,16}$ Therefore, we attempted to synthesize chiral $\mathrm{N}, \mathrm{O}$-type organocatalysts based on inherently chiral calix[4]arenes substituted at the lower rim, and selected Henry reaction between aromatic aldehydes and nitromethane as a model reaction to explore their organocatalytic abilities.

\section{Experimental}

\section{General experimental procedures}

All reactions were conducted under atmosphere without special drying. All chemicals were purchased from commercial sources and used without further purification. Melting points were measured on RY-1G melting point apparatus. Nuclear magnetic resonance (NMR) spectra were performed on Bruker Avance $400\left({ }^{1} \mathrm{H}: 400 \mathrm{MHz}\right.$, $\left.{ }^{13} \mathrm{C}: 101 \mathrm{MHz}\right) . \mathrm{CDCl}_{3}(\delta 7.26 \mathrm{ppm})$, DMSO- $d_{6}(\delta 2.50$, $3.33 \mathrm{ppm})$ or TMS ( $\delta 0.00 \mathrm{ppm})$ was used as an internal standard for ${ }^{1} \mathrm{H}$ NMR spectra, $\mathrm{CDCl}_{3}(\delta 77.00 \mathrm{ppm})$, DMSO- $d_{6}(\delta 39.58 \mathrm{ppm})$ or TMS $(\delta 0.00 \mathrm{ppm})$ was used as an internal standard for ${ }^{13} \mathrm{C}$ NMR spectra. The following abbreviations are used to indicate the multiplicity in NMR spectra: $\mathrm{s}=$ singlet, $\mathrm{d}=$ doublet, $\mathrm{t}=$ triplet, $\mathrm{q}=$ quartet, $\mathrm{m}=$ multiplet, $J$ indicates the NMR coupling constant measured in Hertz. The courses of the reactions were monitored by thin layer chromatography (TLC) using TLC aluminium sheets with silica gel 60 GF254. The 
column chromatography was performed using silica gel 60 . Optical rotations were measured on a Jasco P-2000 digital polarimeter. High-resolution mass spectra (HRMS) were recorded on a 7.0-T (Ionspec, Irvine, CA, USA) Fourier transform ion cyclotron resonance mass spectrometer.

\section{Synthesis}

To a stirred solution of $1 \mathbf{a}$ (or $1 \mathbf{b})^{17}(2.00 \mathrm{~g}, 1.98 \mathrm{mmol})$ in tetrahydrofuran $(30 \mathrm{~mL})$ was added sodium hydride $(0.38 \mathrm{~g}$, $7.9 \mathrm{mmol}$ ) at room temperature. The reaction mixture was heated at reflux for $4 \mathrm{~h}$. Then the 2-methoxyethyl tosylate was added, and the stir was continued for $2 \mathrm{~h}$ and quenched with hydrocholoric acid $\left(2.00 \mathrm{~mol} \mathrm{~L}^{-1}\right)$. The aqueous layer was extracted with dichloromethane, and the combined organic layers were washed with saturated brine, dried with anhydrous sodium sulfate. The solvent was removed under reduced pressure to yield product which was further purified by silica gel column chromatography (eluent $=$ petroleum ether:ethyl acetate, 2:1, v/v). Compound 2a (or $\mathbf{2 b}$ ) was obtained as white power.

\section{$2 a$}

Yield: $1.6 \mathrm{~g}(80 \%)$; m.p. $108-111{ }^{\circ} \mathrm{C} ;[\alpha]_{\mathrm{D}}^{25}=+0.1$ (c $=1.8 \mathrm{~g} / 100 \mathrm{~mL}, \mathrm{CH}_{2} \mathrm{Cl}_{2}$ ); ${ }^{1} \mathrm{H} \mathrm{NMR}\left(400 \mathrm{MHz}, \mathrm{CDCl}_{3}\right.$ ) $\delta 7.63(\mathrm{~d}, 1 \mathrm{H}, J 8.4 \mathrm{~Hz}, \mathrm{NH}), 7.28(\mathrm{~m}, 9 \mathrm{H}, \mathrm{Ar}-\mathrm{H}), 7.13(\mathrm{dd}$, $2 \mathrm{H}, J$ 2.4, $2.4 \mathrm{~Hz}, \mathrm{Ar}-\mathrm{H}$ ), 7.09 (dd, $2 \mathrm{H}, J 2.4,2.4 \mathrm{~Hz}, \mathrm{Ar}-\mathrm{H}$ ), 6.09 (dd, 2H, J 2.4, 2.4 Hz, Ar-H), 6.50 (dd, 2H, J 2.4, $2.4 \mathrm{~Hz}$, Ar-H), 5.23-5.20 (m, 1H, Ph-CH), 4.55 (d, $1 \mathrm{H}, J 15.2 \mathrm{~Hz}$, $\mathrm{O}-\mathrm{CH}_{2}-\mathrm{CO}$ ), 4.11 (d, $1 \mathrm{H}, J 15.2 \mathrm{~Hz}, \mathrm{O}-\mathrm{CH}_{2}-\mathrm{CO}$ ), 4.00-3.07 (m, $14 \mathrm{H}, \mathrm{Ar}-\mathrm{CH}_{2}-\mathrm{Ar}, \mathrm{O}-\mathrm{CH}_{2}-\mathrm{CH}_{2}-\mathrm{CH}_{3}, \mathrm{O}-\mathrm{CH}_{2}-\mathrm{CH}_{2}-\mathrm{O}-\mathrm{CH}_{3}$ ), 3.04 (s, 3H, O- $\left.\mathrm{CH}_{3}\right), 2.46$ (s, 3H, Ar- $\left.-\mathrm{CH}_{3}\right), 1.84(\mathrm{~m}, 2 \mathrm{H}$, $\left.\mathrm{O}-\mathrm{CH}_{2}-\underline{\mathrm{CH}}_{2}-\mathrm{CH}_{3}\right), 1.53$ (d, $\left.3 \mathrm{H}, J 6.8 \mathrm{~Hz}, \mathrm{CH}-\underline{\mathrm{CH}}_{3}\right), 1.36$ (s, 9H, $t-\mathrm{Bu}), 1.32(\mathrm{~s}, 9 \mathrm{H}, t-\mathrm{Bu}), 1.07(\mathrm{~s}, 9 \mathrm{H}, t-\mathrm{Bu}), 1.04$ (s, $9 \mathrm{H}, t-\mathrm{Bu}$ ), 0.98 (t, $3 \mathrm{H}, J 14.8 \mathrm{~Hz}, \mathrm{O}-\mathrm{CH}_{2}-\mathrm{CH}_{2}-\mathrm{CH}_{3}$ ); ${ }^{13} \mathrm{C}$ NMR $\left(101 \mathrm{MHz}, \mathrm{CDCl}_{3}\right) \delta 153.8,153.6,147.2,145.5$, 145.2, 145.0, 144.6, 144.2, 140.8, 135.5, 135.4, 134.0, 131.9, $130.5,129.6,129.3,128.7,128.5,126.1,125.7,125.7,125.4$, 125.1, 125.0, 124.9, 72.8, 71.8, 71.0, 70.4, 58.7, 58.6, 58.3, 37.0, 36.2, 34.4, 34.1, 33.8, 31.7, 31.6, 31.5, 31.4, 31.3, 31.2, 23.8, 21.8, 10.6. HRMS (ESI) $\mathrm{m} / z$, calcd. for $\mathrm{C}_{67} \mathrm{H}_{87} \mathrm{NO}_{8} \mathrm{SNa}^{+}$ $1088.6152[\mathrm{M}+\mathrm{Na}]^{+}$, found $1088.5781[\mathrm{M}+\mathrm{Na}]^{+}$.

\section{2b}

Yield: $1.51 \mathrm{~g}(76 \%) ;$ m.p. $96-98{ }^{\circ} \mathrm{C} ;[\alpha]_{\mathrm{D}}^{25}=-2.4$ (c $\left.=2.0 \mathrm{~g} / 100 \mathrm{~mL}, \mathrm{CH}_{2} \mathrm{Cl}_{2}\right) ;{ }^{1} \mathrm{H} \mathrm{NMR}\left(400 \mathrm{MHz}, \mathrm{CDCl}_{3}\right) \delta$ 7.67 (d, $1 \mathrm{H}, J 8 \mathrm{~Hz}, \mathrm{NH}), 7.28$ (m, 9H, Ar-H), $7.18(\mathrm{dd}, 2 \mathrm{H}$, $J$ 2.4, $2.4 \mathrm{~Hz}, \mathrm{Ar}-\mathrm{H}$ ), 7.08 (dd, 2H, J 2.4, $2.4 \mathrm{~Hz}, \mathrm{Ar}-\mathrm{H}$ ), 6.89 (dd, $2 \mathrm{H}, J 2.4,2.4 \mathrm{~Hz}, \mathrm{Ar}-\mathrm{H}), 6.48$ (dd, 2H, J 2.4, $2.4 \mathrm{~Hz}$, Ar-H), 5.35-5.25 (m, 1H, Ph-CH), 4.46 (d, 1H, J $14.8 \mathrm{~Hz}$, O-CH $-\mathrm{CO}$ ), 4.11 (d, $1 \mathrm{H}, J 14.8 \mathrm{~Hz}, \mathrm{O}-\mathrm{CH}_{2}-\mathrm{CO}$ ), 3.00-4.05 (m, $14 \mathrm{H}, \mathrm{Ar}-\mathrm{CH}_{2}-\mathrm{Ar}, \mathrm{O}-\mathrm{CH}_{2}-\mathrm{CH}_{2}-\mathrm{CH}_{3}, \mathrm{O}-\mathrm{CH}_{2}-\mathrm{CH}_{2}-\mathrm{O}-\mathrm{CH}_{3}$ ), $2.92\left(\mathrm{~s}, 3 \mathrm{H}, \mathrm{O}-\mathrm{CH}_{3}\right), 2.46$ (s, 3H, $\left.\mathrm{Ar}-\mathrm{CH}_{3}\right), 1.92-1.78$ (m, $\left.2 \mathrm{H}, \mathrm{O}-\mathrm{CH}_{2}-\mathrm{CH}_{2}-\mathrm{CH}_{3}\right), 1.54$ (d, 3H, J7.2 Hz, CH- $\left.\underline{-C H}_{3}\right), 1.39$ (s, 9H, $t$-Bu), $1.35(\mathrm{~s}, 9 \mathrm{H}, t-\mathrm{Bu}), 1.05(\mathrm{~d}, 18 \mathrm{H}, 2 t-\mathrm{Bu}), 0.97$ (t, $\left.3 \mathrm{H}, J 14.8 \mathrm{~Hz}, \mathrm{O}-\mathrm{CH}_{2}-\mathrm{CH}_{2}-\mathrm{CH}_{3}\right) ;{ }^{13} \mathrm{C}$ NMR $(101 \mathrm{MHz}$, $\left.\mathrm{CDCl}_{3}\right) \delta 168.6,153.5,153.0,152.5,147.4,145.6,145.5$, 145.3, 145.2, 144.5, 144.4, 135.8, 135.4, 134.9, 134.1, 134.0, 131.6, 131.4, 130.2, 129.8, 129.7, 129.5, 128.9, 128.7, 128.4, $128.0,127.4,127.0,126.8,126.4,126.2,125.8,125.6,70.0$, 69.5, 69.1, 67.7, 67.4, 57.6, 36.9, 36.5, 34.3, 34.2, 33.8, 31.7, 31.6, 31.4, 31.3, 23.8, 21.8, 21.0, 10.7. HRMS (ESI) $\mathrm{m} / z$, calcd. for $\mathrm{C}_{67} \mathrm{H}_{87} \mathrm{NO}_{8} \mathrm{SNa}^{+} 1088.6152[\mathrm{M}+\mathrm{Na}]^{+}$, found $1088.5779[\mathrm{M}+\mathrm{Na}]^{+}$.

To a stirred solution of $\mathbf{2 a}$ (or $\mathbf{2 b})(1.50 \mathrm{~g}, 1.42 \mathrm{mmol}$ ) in butyl alcohol $(10 \mathrm{~mL})$ was added potassium tert-butylate $(2 \mathrm{~g}, 17.8 \mathrm{mmol})$ at room temperature. When the solid dissolved, dimethyl sulfoxide $(1.50 \mathrm{~mL})$ was added and the mixture was heated and stirred at $100{ }^{\circ} \mathrm{C}$ for $1 \mathrm{~h}$. Then water $(0.05 \mathrm{~g}, 2$ equiv.) was added and heated at reflux for $24 \mathrm{~h}$. The reaction mixture was evaporated to yield black oily liquid and redissolved in hydrochloric acid $(30 \mathrm{~mL})$ and dichloromethane $(30 \mathrm{~mL})$. The aqueous layer was extracted with dichloromethane $(2 \times 30 \mathrm{~mL})$, and the combined organic layers were washed with brine, dried with anhydrous sodium sulfate, and evaporated to yield brown oily liquid. The product $\mathbf{3 a}$ (or $\mathbf{3 b}$ ) was further purified by silica gel column chromatography (eluent $=$ petroleum ether:ethyl acetate, $3: 1, \mathrm{v} / \mathrm{v}$ ).

$3 a$

Yield: $0.81 \mathrm{~g}(54 \%)$; m.p. $110-113{ }^{\circ} \mathrm{C} ;[\alpha]_{\mathrm{D}}^{25}=+7.4$ (c $\left.=6 \mathrm{~g} / 100 \mathrm{~mL}, \mathrm{CH}_{2} \mathrm{Cl}_{2}\right) ;{ }^{1} \mathrm{H}$ NMR $\left(400 \mathrm{MHz}, \mathrm{CDCl}_{3}\right.$ ) $\delta 8.15(\mathrm{~s}, 1 \mathrm{H}, \mathrm{COOH}), 7.25(\mathrm{~s}, 1 \mathrm{H}, \mathrm{OH}), 7.09(\mathrm{~d}, 1 \mathrm{H}, J 2 \mathrm{~Hz}$, Ar-H), 6.99 (s, 2H, Ar-H), 6.97-6.90 (m, 3H, Ar-H), 6.86 (dd, $2 \mathrm{H}, J 2.4,2.4 \mathrm{~Hz}, \mathrm{Ar}-\mathrm{H}), 4.85$ (d, 1H, O-CH ${ }_{2}-\mathrm{CO}, J 16.0 \mathrm{~Hz}$ ), 4.50-3.60 (m, $\left.11 \mathrm{H}, \mathrm{Ar}-\mathrm{CH}_{2}-\mathrm{Ar}, \mathrm{O}-\mathrm{CH}_{2}-\mathrm{CH}_{2}-\mathrm{O}, \mathrm{O}-\mathrm{CH}_{2}-\mathrm{CO}\right)$, 3.46 (s, 3H, O- $\left.\mathrm{CH}_{3}\right), 3.38-3.21$ (m, $4 \mathrm{H}, \mathrm{O}-\mathrm{CH}_{2}-\mathrm{CH}_{2}-\mathrm{CH}_{3}$, $\left.\mathrm{O}-\mathrm{CH}_{2}-\mathrm{CH}_{2}-\mathrm{O}\right), 2.10$ (m, $\left.2 \mathrm{H}, \mathrm{O}-\mathrm{CH}_{2}-\mathrm{CH}_{2}-\mathrm{CH}_{3}\right), 1.18$ (d, $18 \mathrm{H}, 2 t-\mathrm{Bu}), 1.14(\mathrm{~s}, 9 \mathrm{H}, t-\mathrm{Bu}), 1.08(\mathrm{t}, 3 \mathrm{H}, J 14.8 \mathrm{~Hz}$, $\left.\mathrm{O}-\mathrm{CH}_{2}-\mathrm{CH}_{2}-\mathrm{CH}_{3}\right), 1.04$ (s, 9H, $t$-Bu); ${ }^{13} \mathrm{C}$ NMR $(101 \mathrm{MHz}$, $\left.\mathrm{CDCl}_{3}\right) \delta 171.1,151.3,149.2,147.1,146.8,146.0,142.5$, 134.0, 132.8, 129.2, 126.4, 125.9, 125.8, 125.7, 125.6, 125.5, 125.0, 78.9, 74.3, 71.6, 71.5, 58.8, 34.1, 32.3, 31.5, 31.4, $31.3,31.2,30.5,22.8,10.3$. HRMS (ESI) $\mathrm{m} / z$, calcd. for $\mathrm{C}_{52} \mathrm{H}_{70} \mathrm{O}_{7} \mathrm{Na}^{+} 806.5122[\mathrm{M}+\mathrm{Na}]^{+}$, found $829.5014[\mathrm{M}+\mathrm{Na}]^{+}$.

\section{3b}

Yield: 0.90 g (60\%); m.p. $110-113{ }^{\circ} \mathrm{C} ;[\alpha]_{D}^{25}=-7.5$ (c $=4.5 \mathrm{~g} / 100 \mathrm{~mL}, \mathrm{CH}_{2} \mathrm{Cl}_{2}$ ). The relative spectroscopic data were identical to those of $\mathbf{3 a}$. 
To a stirring solution of $\mathbf{3 a}$ ( (or $\mathbf{3 b}$ ) $(0.50 \mathrm{~g}, 0.63 \mathrm{mmol})$ in dichloromethane was added $O$-benzotriazole$N, N, N$ ', $N$ '-tetramethyl-uronium-hexafluorophosphate $(0.36 \mathrm{~g}, 0.95 \mathrm{mmol})$ followed by the addition of 4-dimethyl aminopyridine $(0.05 \mathrm{~g}, 0.41 \mathrm{mmol})$. The reaction mixture was stirred at room temperature for half an hour. $\mathrm{N}, \mathrm{N}$-Diethylamine was added and stirring was continued for $6 \mathrm{~h}$ at room temperature. The reaction mixture was quenched with dilute hydrochloric acid and the aqueous layer was extracted with dichloromethane $(2 \times 30 \mathrm{~mL})$, and the combined organic layers were washed with brine, dried with anhydrous sodium sulfate, and evaporated to dryness. The product $\mathbf{4 a}$ (or $\mathbf{4 b}$ ) was further purified by silica gel column chromatography (eluent $=$ petroleum ether:ethyl acetate, $3: 1, \mathrm{v} / \mathrm{v})$.

\section{4a}

Yield: $0.32 \mathrm{~g}(65 \%)$; m.p. $96-98{ }^{\circ} \mathrm{C} ;[\alpha]_{\mathrm{D}}^{25}=+5.8$ (c $\left.=2.2 \mathrm{~g} / 100 \mathrm{~mL}, \mathrm{CH}_{2} \mathrm{Cl}_{2}\right) ;{ }^{1} \mathrm{H}$ NMR $(400 \mathrm{MHz}, \mathrm{DMSO})$ $\delta 7.26(\mathrm{~s}, 1 \mathrm{H}, \mathrm{OH}), 7.04$ (dd, 2H, J 2.4, $2.4 \mathrm{~Hz}, \mathrm{Ar}-\mathrm{H})$, 6.98 (dd, 2H, J 2.4, $2.4 \mathrm{~Hz}, \mathrm{Ar}-\mathrm{H}$ ), 6.66 (dd, 2H, J 2.4, $2.4 \mathrm{~Hz}, \mathrm{Ar}-\mathrm{H}), 6.53$ (dd, 2H, J 2.4, 2.4 Hz, Ar-H), 4.72 (d, $1 \mathrm{H}, J 15.6 \mathrm{~Hz}, \mathrm{O}-\mathrm{CH}_{2}-\mathrm{CO}$ ), $4.52-4.40$ (m, 3H, O- $\mathrm{CH}_{2}-\mathrm{CO}$, Ar- $\mathrm{CH}_{2}-\mathrm{Ar}$ ), 4.37 (d, $1 \mathrm{H}, \mathrm{Ar}-\mathrm{CH}_{2}-\mathrm{Ar}, J 12.0 \mathrm{~Hz}$ ), 4.29 (d, $1 \mathrm{H}, \mathrm{Ar}-\mathrm{CH}_{2}-\mathrm{Ar}, J 12.0 \mathrm{~Hz}$ ), 3.12 (m, $4 \mathrm{H}, \mathrm{Ar}-\mathrm{CH}_{2}-\mathrm{Ar}$ ), 3.90$3.70\left(\mathrm{~m}, 2 \mathrm{H}, \mathrm{O}-\mathrm{CH}_{2}-\mathrm{CH}_{2}-\mathrm{O}\right), 3.50\left(\mathrm{~m}, 4 \mathrm{H}, \mathrm{O}-\mathrm{CH}_{2}-\mathrm{CH}_{2}-\mathrm{O}\right.$, $\mathrm{O}-\mathrm{CH}_{2}-\mathrm{CH}_{2}-\mathrm{CH}_{3}$ ), 3.45 (s, 3H, O- $\mathrm{CH}_{3}$ ), 3.25-3.18 (m, $4 \mathrm{H}$, $\mathrm{N}-\mathrm{CH}_{2}-\mathrm{CH}_{3}$ ), 2.05-1.93 (m, $\left.2 \mathrm{H}, \mathrm{O}-\mathrm{CH}_{2}-\mathrm{CH}_{2}-\mathrm{CH}_{3}\right), 1.32-$ $1.15\left(\mathrm{~m}, 24 \mathrm{H}, t-\mathrm{Bu}, \mathrm{N}-\mathrm{CH}_{2}-\mathrm{CH}_{3}\right), 1.07(\mathrm{t}, 3 \mathrm{H}, J 14.8 \mathrm{~Hz}$, $\mathrm{O}-\mathrm{CH}_{2}-\mathrm{CH}_{2}-\mathrm{CH}_{3}$ ), 0.93 (s, 9H, $t$-Bu), 0.83 (s, 9H, $t$-Bu); ${ }^{13} \mathrm{C} \mathrm{NMR}\left(101 \mathrm{MHz}, \mathrm{CDCl}_{3}\right) \delta 167.5,152.8,152.7,151.9$, 145.0, 144.7, 144.6, 144.4, 139.8, 134.2, 134.0, 133.0, $132.6,132.5,132.4,127.7,127.4,125.3,125.2,125.1$, 124.6, 124.5, 77.0, 73.1, 72.1, 70.7, 58.0, 33.6, 33.4, 33.3, $31.4,31.2,30.9,30.8,22.7,14.1,12.8,10.5$. HRMS (ESI) $\mathrm{m} / z$, calcd. for $\mathrm{C}_{56} \mathrm{H}_{79} \mathrm{NO}_{6} \mathrm{Na}^{+} 861.5907[\mathrm{M}+\mathrm{Na}]^{+}$, found $884.5804[\mathrm{M}+\mathrm{Na}]^{+}$.

\section{$4 b$}

Yield: $0.35 \mathrm{~g}(71 \%)$; m.p. $96-98{ }^{\circ} \mathrm{C} ;[\alpha]_{\mathrm{D}}^{25}=-5.8$ (c $=7.5 \mathrm{~g} / 100 \mathrm{~mL}, \mathrm{CH}_{2} \mathrm{Cl}_{2}$ ). The relative spectroscopic data were identical to those of $\mathbf{4 a}$.

To a solution of $\mathbf{4 a}$ (or $\mathbf{4 b})(0.20 \mathrm{~g}, 0.24 \mathrm{mmol})$ in tetrahydrofuran $(10 \mathrm{~mL})$ was slowly added lithium aluminum hydride $(0.18 \mathrm{~g}, 4.72 \mathrm{mmol})$ at $0{ }^{\circ} \mathrm{C}$. After the mixture was stirred for $5 \mathrm{~h}$ at room temperature, the resulting mixture was filtered and the filtrate was concentrated. The reaction mixture was redissolved in hydrochloric acid $(10 \mathrm{~mL})$ and dichloromethane $(10 \mathrm{~mL})$. The aqueous layer was extracted with dichloromethane $(2 \times 10 \mathrm{~mL})$, and the combined organic layers were washed with brine, dried with anhydrous sodium sulfate, and evaporated to yield white liquid. The product $\mathbf{5 a}$ (or $\mathbf{5 b}$ ) was further purified by silica gel column chromatography (eluent $=$ petroleum ether:ethyl acetate, $2: 1, \mathrm{v} / \mathrm{v}$ ).

\section{$5 a$}

Yield: $0.07 \mathrm{~g}(33 \%)$; m.p. $78-81{ }^{\circ} \mathrm{C} ;[\alpha]_{\mathrm{D}}^{25}=+2.8$ ( $\left.\mathrm{c}=7.5 \mathrm{~g} / 100 \mathrm{~mL}, \mathrm{CH}_{2} \mathrm{Cl}_{2}\right) ;{ }^{1} \mathrm{H} \mathrm{NMR}\left(400 \mathrm{MHz}, \mathrm{CDCl}_{3}\right)$ $\delta 7.13$ (s, 2H, Ar-H), 7.05 (dd, 2H, J 2.4, $2.4 \mathrm{~Hz}, \mathrm{Ar}-\mathrm{H}$ ), 6.52-6.50 (m, 4H, Ar-H), 5.75 (s, 1H, OH), 4.40-4.24 (m, $4 \mathrm{H}, \mathrm{Ar}-\mathrm{CH}_{2}-\mathrm{Ar}$ ), 4.22-4.05 (m, 4H, Ar- $\mathrm{CH}_{2}-\mathrm{Ar}$ ), 3.92 (m, $2 \mathrm{H}, \mathrm{O}-\mathrm{CH}_{2}-\mathrm{CH}_{2}-\mathrm{O}$ ), 3.87-3.78 (m, $1 \mathrm{H}, \mathrm{O}-\mathrm{CH}_{2}-\mathrm{CH}_{2}-\mathrm{N}$ ), 3.77-3.67 (m, $\left.1 \mathrm{H}, \mathrm{O}-\mathrm{CH}_{2}-\mathrm{CH}_{2}-\mathrm{N}\right), 3.48\left(\mathrm{~s}, 1 \mathrm{H}, \mathrm{O}-\mathrm{CH}_{3}\right), 3.29-$ $3.13\left(\mathrm{~m}, 4 \mathrm{H}, \mathrm{O}-\mathrm{CH}_{2}-\mathrm{CH}_{2}-\mathrm{O}, \mathrm{O}-\mathrm{CH}_{2}-\mathrm{CH}_{2}-\mathrm{CH}_{3}\right), 3.04$ (m, $\left.2 \mathrm{H}, \mathrm{O}-\mathrm{CH}_{2}-\mathrm{CH}_{2}-\mathrm{N}\right), 2.62\left(\mathrm{~m}, 4 \mathrm{H}, \mathrm{N}-\mathrm{CH}_{2}-\mathrm{CH}_{3}\right), 2.08-1.84$ $\left(\mathrm{m}, 2 \mathrm{H}, \mathrm{O}-\mathrm{CH}_{2}-\mathrm{CH}_{2}-\mathrm{CH}_{3}\right), 1.33(\mathrm{~s}, 9 \mathrm{H}, t-\mathrm{Bu}), 1.32(\mathrm{~s}, 9 \mathrm{H}$, $t$ - $\mathrm{Bu}), 1.11\left(\mathrm{t}, 3 \mathrm{H}, J 14.2 \mathrm{~Hz}, \mathrm{O}-\mathrm{CH}_{2}-\mathrm{CH}_{2}-\mathrm{CH}_{3}\right), 1.06(\mathrm{t}, 6 \mathrm{H}$, $\left.J 14.4 \mathrm{~Hz}, \mathrm{~N}-\mathrm{CH}_{2}-\mathrm{CH}_{3}\right), 0.83(\mathrm{~s}, 9 \mathrm{H}, t-\mathrm{Bu}), 0.81(\mathrm{~s}, 9 \mathrm{H}, t-\mathrm{Bu})$; ${ }^{13} \mathrm{C}$ NMR $\left(101 \mathrm{MHz}, \mathrm{CDCl}_{3}\right) \delta 151.7,150.7,145.8,145.2$, 135.9, 135.8, 132.2, 131.9, 131.8, 129.2, 129.0, 125.7, 125.1, 125.0, 124.9, 124.8, 124.7, 78.1, 74.1, 71.4, 71.1, 58.9, 52.5, 47.8, 34.2, 33.8, 33.7, 33.6, 31.8, 31.7, 31.4, 31.3, 31.1, 31.0, 23.4, 11.8, 10.9. HRMS (ESI) $\mathrm{m} / 2$, calcd. for $\mathrm{C}_{56} \mathrm{H}_{81} \mathrm{NO}_{5} \mathrm{H}^{+}$ $848.6115[\mathrm{M}+\mathrm{H}]^{+}$, found $848.6186[\mathrm{M}+\mathrm{H}]^{+}$.

\section{$5 b$}

Yield: 0.08 g (38\%); m.p. $78-81{ }^{\circ} \mathrm{C} ;[\alpha]_{\mathrm{D}}^{25}=-2.8$ (c $=7.5 \mathrm{~g} / 100 \mathrm{~mL}, \mathrm{CH}_{2} \mathrm{Cl}_{2}$ ). The relative spectroscopic data were identical to those of $\mathbf{5 a}$.

\section{General Henry reaction procedure}

$6 \mathrm{mmol}$ nitromethane and $0.5 \mathrm{mmol} \%$ inherently chiral calix[4]arene catalyst were mixed and stirred in $4 \mathrm{~mL}$ solvent under nitrogen at an experimental temperature. After 15 minutes, $0.6 \mathrm{mmol}$ aldehyde was added, and the mixture was continuously stirred. After completion, the reaction mixture was removed under reduced pressure. The crude residue was loaded directly onto a silica gel column and purified by flash chromatography as described below.

\section{2-Nitro-1-(4-nitrophenyl)ethanol}

The title compound was prepared according to the General experimental procedures section and purified by column chromatographic purification $\left(\mathrm{CH}_{2} \mathrm{Cl}_{2}\right)$, giving a white solid. ${ }^{1} \mathrm{H}$ NMR $\left(400 \mathrm{MHz}, \mathrm{CDCl}_{3}\right) \delta 3.20(\mathrm{~s}, 1 \mathrm{H}$, - $\mathrm{OH}$ ), 4.53-4.69 (m, 2H, - $\mathrm{CH}_{2} \mathrm{NO}_{2}$ ), 5.62 (ddd, $J$ 4.0, 4.1, $8.1 \mathrm{~Hz}, 1 \mathrm{H},-\mathrm{C} \underline{\mathrm{HOH}}), 7.65$ (d, J $8.7 \mathrm{~Hz}, 2 \mathrm{H}, \mathrm{ArH}), 8.27$ (d, $J 8.7 \mathrm{~Hz}, 2 \mathrm{H}, \mathrm{ArH}) ;{ }^{13} \mathrm{C} \mathrm{NMR}\left(101 \mathrm{MHz}, \mathrm{CDCl}_{3}\right) \delta 69.9$, 80.6, 124.2, 126.9, 144.9, 148.2. 
2-Nitro-1-(4-cyanophenyl)ethanol

The title compound was prepared according to the General experimental procedures section and purified by column chromatographic purification $\left(\mathrm{CH}_{2} \mathrm{Cl}_{2}\right)$, giving a white powder. ${ }^{1} \mathrm{H}$ NMR $\left(400 \mathrm{MHz}, \mathrm{CDCl}_{3}\right) \delta 3.31(\mathrm{~s}, 1 \mathrm{H}$, - $\mathrm{OH}$ ), 4.52-4.62 (m, 2H, $-\mathrm{CH}_{2} \mathrm{NO}_{2}$ ), 5.55 (ddd, J 4.1, 4.2, $8.5 \mathrm{~Hz}, 1 \mathrm{H},-\mathrm{C} \underline{\mathrm{HOH}}), 7.57$ (d, J $8.3 \mathrm{~Hz}, 2 \mathrm{H}, \mathrm{ArH}), 7.70$ (d, $J 8.3 \mathrm{~Hz}, 2 \mathrm{H}, \mathrm{ArH}) ;{ }^{13} \mathrm{C} \mathrm{NMR}\left(101 \mathrm{MHz}, \mathrm{CDCl}_{3}\right) \delta 70.1$, 80.6, 112.9, 118.1, 126.7, 132.8, 143.0.

\section{2-Nitro-1-(4-fluorophenyl)ethanol}

The title compound was prepared according to the General experimental procedures section and purified by column chromatographic purification $\left(\mathrm{CH}_{2} \mathrm{Cl}_{2}\right)$, giving a colorless oil. ${ }^{1} \mathrm{H}$ NMR (400 MHz, $\left.\mathrm{CDCl}_{3}\right) \delta 2.88(\mathrm{~s}, 1 \mathrm{H}$, $-\mathrm{OH}$ ), 4.50 (dd, J 3.1, $13.4 \mathrm{~Hz}, 1 \mathrm{H},-\mathrm{CH}_{2} \mathrm{NO}_{2}$ ), 4.59 (dd, $J$ 9.4, $13.4 \mathrm{~Hz}, 1 \mathrm{H},-\mathrm{CH}_{2} \mathrm{NO}_{2}$ ), 5.45 (dd, J 3.1, $9.4 \mathrm{~Hz}$, 1H, -C트), 7.06-7.12 (m, 2H, ArH), 7.37-7.42 (m, 2H, $\mathrm{ArH}) ;{ }^{13} \mathrm{C} \mathrm{NMR}\left(101 \mathrm{MHz}, \mathrm{CDCl}_{3}\right) \delta 70.3,81.1,116.0$, $127.8,133.8,162.9$.

\section{2-Nitro-1-(2-nitrophenyl)ethanol}

The title compound was prepared according to the General experimental procedures section and purified by column chromatographic purification (petroleum ether:ethyl acetate, 5:1 v/v), giving a yellow oil. ${ }^{1} \mathrm{H}$ NMR $\left(400 \mathrm{MHz}, \mathrm{CDCl}_{3}\right) \delta 3.52$ (s, 1H, -OH), 4.56 (dd, J 13.9, $\left.8.8 \mathrm{~Hz}, 1 \mathrm{H},-\mathrm{CH}_{2} \mathrm{NO}_{2}\right), 4.84(\mathrm{dd}, J 13.9,2.2 \mathrm{~Hz}, 1 \mathrm{H}$, $-\mathrm{CH}_{2} \mathrm{NO}_{2}$ ), 6.01 (ddd, J 2.2, 4.2, 8.8 Hz, 1H, - $\mathrm{CHOH}$ ), 7.517.59 (m, 1H, ArH), 7.71-7.79 (m, 1H, ArH), 7.90-7.96 (m, 1H, ArH), 8.02-8.07 (m, 1H, ArH); ${ }^{13} \mathrm{C} \mathrm{NMR} \mathrm{(101} \mathrm{MHz,}$ $\left.\mathrm{CDCl}_{3}\right) \delta 69.9,80.6,124.2,126.9,144.9,148.2$.

\section{2-Nitro-1-(3-nitrophenyl)ethanol}

The title compound was prepared according to the General experimental procedures section and purified by column chromatographic purification (petroleum ether:ethyl acetate, 5:1 v/v), giving a brown oil. ${ }^{1} \mathrm{H}$ NMR (400 MHz, $\left.\mathrm{CDCl}_{3}\right) \delta 3.39$ (s, $\left.1 \mathrm{H},-\mathrm{OH}\right), 4.58-4.67$ (m, $2 \mathrm{H},-\mathrm{CH}_{2} \mathrm{NO}_{2}$ ), 5.60 (dd, J3.3, 9.3 Hz, 1H, -CㅌH), 7.56-7.66 (m, 1H, ArH), 7.75-7.79 (m, 1H, ArH), 8.18-8.24 (m, 1H, ArH), 8.28-8.36 $(\mathrm{m}, 1 \mathrm{H}, \mathrm{ArH}) ;{ }^{13} \mathrm{C}$ NMR $\left(101 \mathrm{MHz}, \mathrm{CDCl}_{3}\right) \delta 71.8,83.2$, 123.4, 127.0, 130.3, 137.6, 140.4, 147.6.

\section{2-Nitro-1-(3-chlorophenyl)ethanol}

The title compound was prepared according to the General experimental procedures section and purified by column chromatographic purification (petroleum ether:ethyl acetate, 5:1 v/v), giving a dark yellow oil. ${ }^{1} \mathrm{H} \mathrm{NMR}\left(400 \mathrm{MHz}, \mathrm{CDCl}_{3}\right) \delta 3.01$ (s, 1H, -OH), 4.48-4.60 (m, 2H, $-\mathrm{CH}_{2} \mathrm{NO}_{2}$ ), 5.44 (dd, J 3.9, $13.2 \mathrm{~Hz}, 1 \mathrm{H},-\mathrm{C} \underline{\mathrm{HOH}}$ ),
7.24-7.29 (m, 2H, ArH), 7.31-7.36 (m, 1H, ArH), 7.42 (s, $1 \mathrm{H}, \mathrm{ArH}) ;{ }^{13} \mathrm{C} \mathrm{NMR}\left(101 \mathrm{MHz}, \mathrm{CDCl}_{3}\right) \delta 71.8,84.2,128.3$, $129.3,130.5,131.2,134.2,146.3$.

\section{2-Nitro-1-(4-methoxyphenyl)ethanol}

The title compound was prepared according to the General experimental procedures section and purified by column chromatographic purification (petroleum ether:ethyl acetate, 3:1 v/v) gave a yellow oil. ${ }^{1} \mathrm{H}$ NMR $\left(400 \mathrm{MHz}, \mathrm{CDCl}_{3}\right) \delta 2.76(\mathrm{~s}, 1 \mathrm{H},-\mathrm{OH}), 3.81(\mathrm{~s}, 3 \mathrm{H}$, $\left.-\mathrm{CH}_{3} \mathrm{O}\right), 4.48\left(\mathrm{dd}, J 2.9,13.2 \mathrm{~Hz}, 1 \mathrm{H},-\mathrm{CH}_{2} \mathrm{NO}_{2}\right), 4.60$ (dd, $J$ 9.6, $13.2 \mathrm{~Hz}, 1 \mathrm{H},-\mathrm{CH}_{2} \mathrm{NO}_{2}$ ), 5.40 (ddd, $J$ 2.9, 4.2, $9.6 \mathrm{~Hz}, 1 \mathrm{H},-\mathrm{C} \underline{\mathrm{HOH}}), 6.92$ (d, J 8.7 Hz, 2H, ArH), 7.32 (d, $J 8.7 \mathrm{~Hz}, 2 \mathrm{H}, \mathrm{ArH}) ;{ }^{13} \mathrm{C} \mathrm{NMR}\left(101 \mathrm{MHz}, \mathrm{CDCl}_{3}\right) \delta 69.9$, 80.6, 124.2, 126.9, 144.9, 148.2.

\section{2-Nitro-1-(2-methylphenyl)ethanol}

The title compound was prepared according to the General experimental procedures section and purified by column chromatographic purification (petroleum ether:ethyl acetate, 10:1 v/v), giving a dark yellow oil. ${ }^{1} \mathrm{H}$ NMR (400 MHz, $\left.\mathrm{CDCl}_{3}\right) \delta 2.38\left(\mathrm{~s}, 3 \mathrm{H},-\mathrm{CH}_{3}\right), 2.69$ (s, 1H, -OH), 4.41-4.45 (dd, J 2.8, $13.2 \mathrm{~Hz}, 1 \mathrm{H},-\mathrm{C} \underline{\mathrm{HOH}}$ ), 4.51-4.57 (dd, J 9.6, $13.2 \mathrm{~Hz}, 1 \mathrm{H},-\mathrm{CHOH}), 5.44$ (m, 1H,

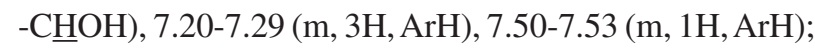
${ }^{13} \mathrm{C}$ NMR $\left(101 \mathrm{MHz}, \mathrm{CDCl}_{3}\right) \delta 10.1,68.1,80.4,125.8$, $127.0,128.9,131.1,134.7,136.5$.

\section{Results and Discussion}

\section{Synthesis}

As shown in Scheme 1, starting from a pair of optically pure inherently chiral calix[4]arene diastereomers 1a and 1b reported by Kalchenko and co-workers, ${ }^{17}$ the alkylation of their last hydroxy groups with 2-methoxyethyl tosylate readily afforded $\mathbf{2} \mathbf{a}$ and $\mathbf{2} \mathbf{b}$ in high yields, respectively. Acids $\mathbf{3 a}$ and $\mathbf{3 b}$ were obtained in moderate yields after the removal of $p$-toluenesulfonyl and phenylethylamide of $\mathbf{2} \mathbf{a}$ and $\mathbf{2} \mathbf{b}$ with $t$-BuOK in a mixed solvent, respectively. Diethylamine was coupled with $\mathbf{3 a}$ and $\mathbf{3 b}$, respectively, in dicholomethane using 2-(1H-benzotriazol-1-yl)-1,1,3,3-tetramethyluronium hexafluorophosphate (HBTU) and 4-dimethylaminopyridine (DMAP) to produce amides $\mathbf{4 a}$ and $\mathbf{4 b}$. The reduction of $\mathbf{4 a}$ and $\mathbf{4 b}$ with $\mathrm{LiAlH}_{4}$ in THF gave novel $N, O$-type compounds $\mathbf{5} \mathbf{a}$ and $\mathbf{5} \mathbf{b}$, respectively. The structures of $\mathbf{2} \mathbf{a}$ and $\mathbf{2 b}, \mathbf{3} \mathbf{a}$ and $\mathbf{3 b}, \mathbf{4 a}$ and $\mathbf{4 b}$, and $\mathbf{5 a}$ and $\mathbf{5 b}$ were confirmed by their ${ }^{1} \mathrm{H}$ NMR, ${ }^{13} \mathrm{C}$ NMR and MS spectra. Their cone or partial cone conformation is clearly shown in the bridging methylene signals of their ${ }^{13} \mathrm{C}$ NMR, respectively. ${ }^{18}$ Since 1a and $\mathbf{1 b}$ have been confirmed as a pair of diastereomers 
by Kalchenko, ${ }^{17}$ it is undoubtedly deduced that $\mathbf{2 a}$ and 2b are a pair of diastereomers, and $\mathbf{3 a}\left([\alpha]_{D}^{25}=+7.4\right)$ and $\mathbf{3 b}\left([\alpha]_{\mathrm{D}}^{25}=-7.5\right), \mathbf{4 a}\left([\alpha]_{\mathrm{D}}^{25}=+5.8\right)$ and $\mathbf{4 b}\left([\alpha]_{\mathrm{D}}^{25}=-5.8\right)$, and $\mathbf{5 a}\left([\alpha]_{\mathrm{D}}^{25}=+2.8\right)$ and $\mathbf{5 b}\left([\alpha]_{\mathrm{D}}^{25}=-2.8\right)$ are three pairs of enantiomers, respectively.

\section{Asymmetric catalysis}

The catalytic abilities of $\mathbf{5 a}$ and $\mathbf{5 b}$ were explored as chiral organocatalysts for Henry (nitroaldol) reaction between 4-nitrobenzaldehyde and nitromethane. The effect of solvent on the reaction was firstly examined at room temperature. The results from the common different solvents were summarized in Table 1. It can be seen that the novel $\mathrm{N}, \mathrm{O}$-type organocatalysts $\mathbf{5 a}$ and $\mathbf{5 b}$ showed an ability to induce enantioselectivity more or less in different solvents. We were delighted to find that an amount of $5 \mathrm{~mol} \%$ catalysts showed sufficient to achieve the reaction. The reaction in $\mathrm{CH}_{3} \mathrm{CN}$ can not afford the desired product in $48 \mathrm{~h}$ (Table 1, entry 5). The yield $(30 \%)$ and enantioselectivity (1.6\% ee) using $\mathbf{5 b}$ were comparably low in THF in $48 \mathrm{~h}$ (Table 1, entry 6). However, the excellent yields and notable enantioselectivities were obtained in $6 \mathrm{~h}$ when $\mathrm{H}_{2} \mathrm{O}$ was added into $\mathrm{CH}_{3} \mathrm{CN}$ and THF, respectively (Table 1, entries 1-4). The yields were not remarkably improved but the enantioselectivities were slightly enhanced in $6 \mathrm{~h}$ when $\mathrm{H}_{2} \mathrm{O}$ was added into EtOH (Table 1, entries 7 and 8 versus entries 9 and 10). The highest enantioselectivity with $7.5 \%$ ee was achieved in a mixed protic solvent $\left(\mathrm{EtOH} / \mathrm{H}_{2} \mathrm{O}, 3: 1\right.$, v/v) although the yield was not very high (54\%) (Table 1, entry 7). These catalytic results showed that protic solvents $\left(\mathrm{H}_{2} \mathrm{O}\right.$ and EtOH) are more beneficial to Henry reaction with higher yield, better enantioselectivity and shorter time than aprotic solvents $\left(\mathrm{CH}_{3} \mathrm{CN}\right.$ and THF). It should be noted that the absolute configuration of major isomers can be puzzlingly inverted along solvent change. The absolute configuration of major isomers using $\mathbf{5 a}$ is always $S$. However, that of major isomers using $\mathbf{5 b}$ is $R$ in $\mathrm{THF} / \mathrm{H}_{2} \mathrm{O}$, THF and EtOH (Table 1, entries 4, 6 and 10), and $S$ in $\mathrm{CH}_{3} \mathrm{CN} /$ $\mathrm{H}_{2} \mathrm{O}$ and $\mathrm{EtOH} / \mathrm{H}_{2} \mathrm{O}$ (Table 1, entries 2, 8 and 12). The absolute configuration inversion of major isomers along solvent change ever took place in the catalytic asymmetric addition of diethylzinc to benzaldehyde with inherently

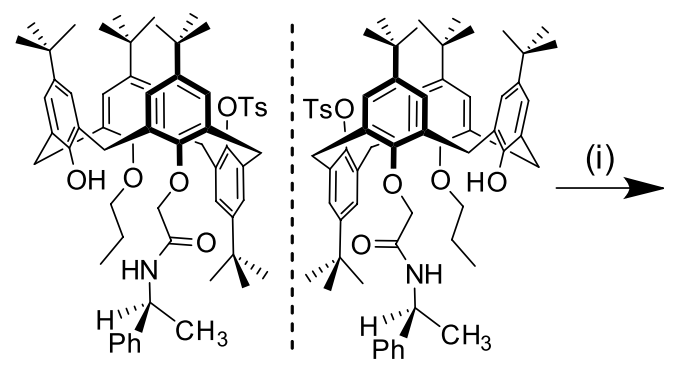

$1 \mathrm{a}$

$1 b$

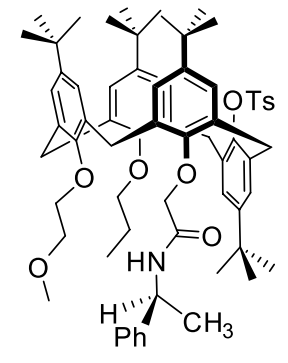

2a

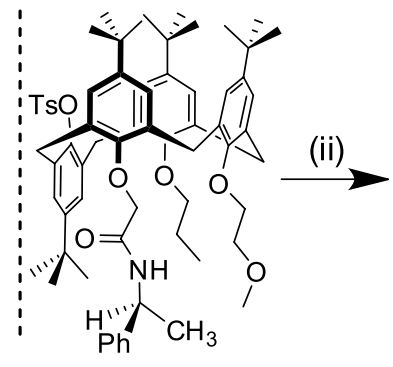

2b

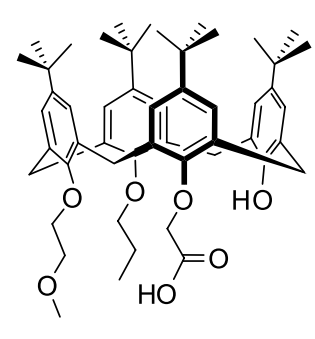

3a

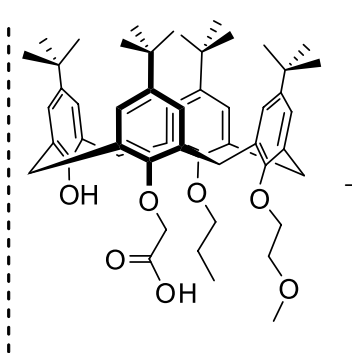

3b

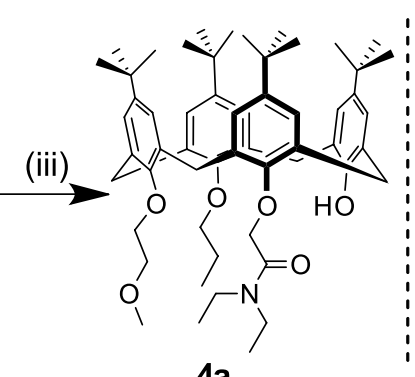

4a

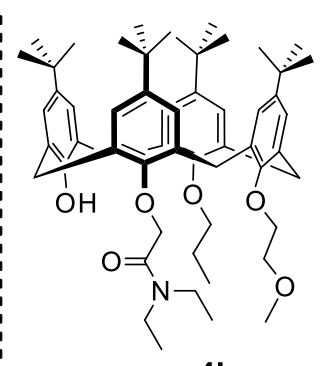

4b
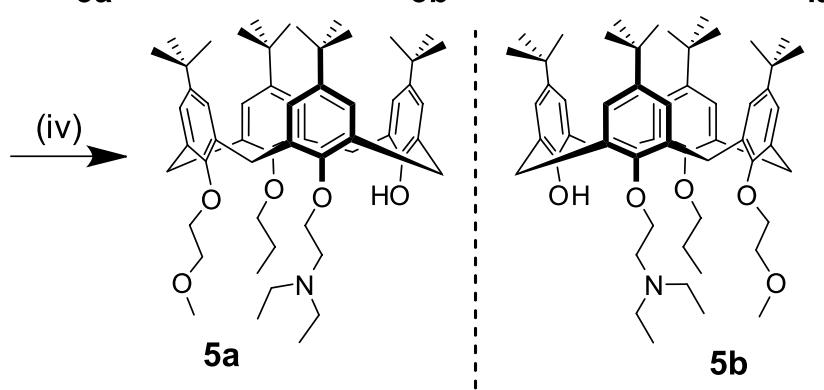

(i) $\mathrm{TsOCH}_{2} \mathrm{CH}_{2} \mathrm{OCH}_{3} / \mathrm{NaH}$, THF; (ii) $t$-BuOK, $\mathrm{H}_{2} \mathrm{O} / \mathrm{BuOH} / \mathrm{DMSO}$; (iii) $\mathrm{HNEt}_{2}, \mathrm{HBTU} \mathrm{CH}_{2} \mathrm{Cl}_{2}$; (iv) $\mathrm{LiAlH}_{4} / \mathrm{THF}$.

Scheme 1. Synthesis of inherently chiral calix[4]arenes substituted at the lower rim. 
Table 1. Optimization of the reaction conditions

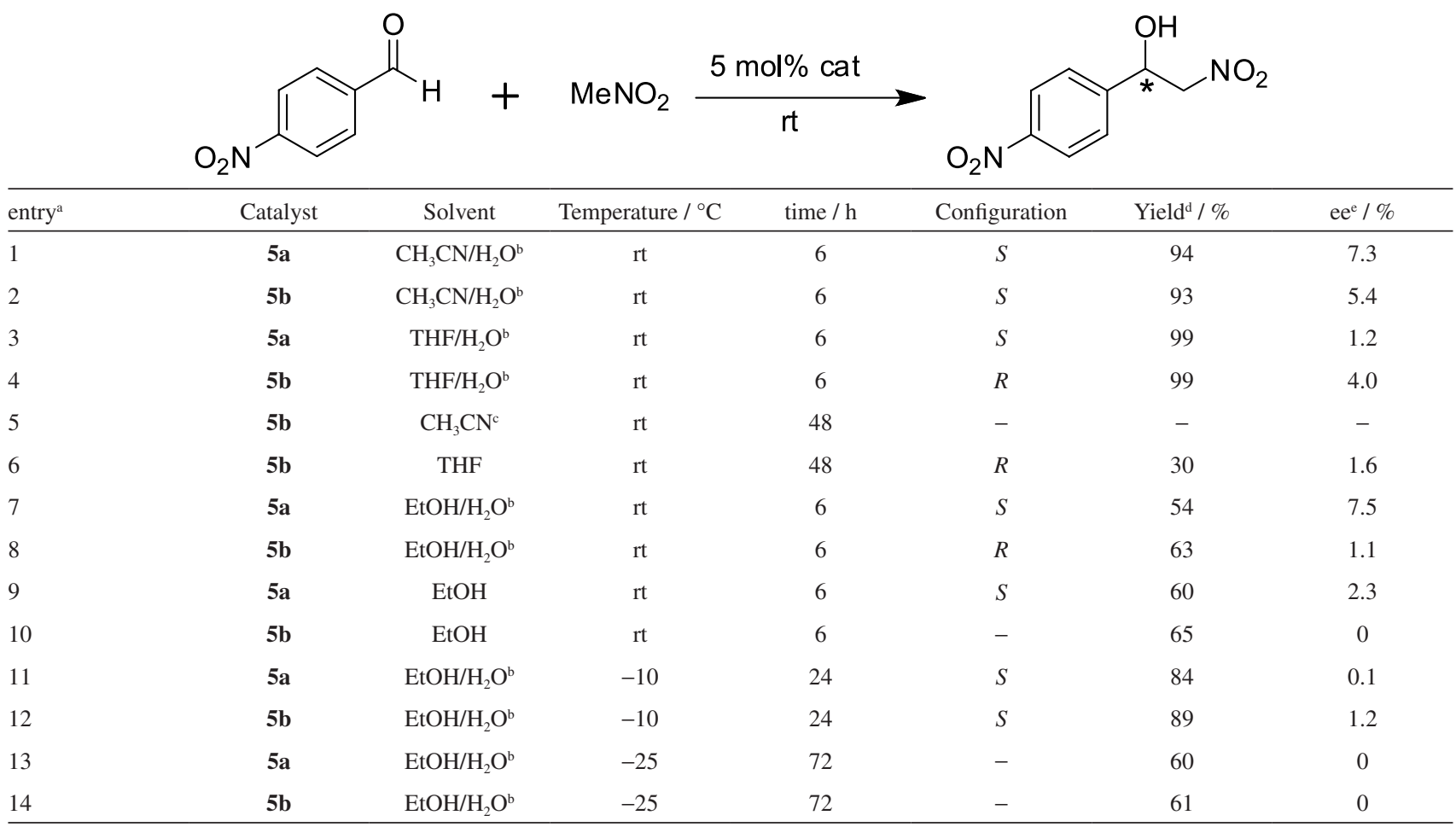

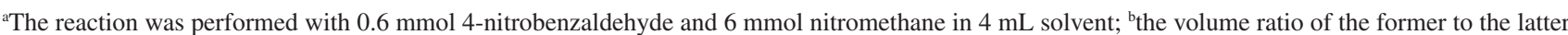

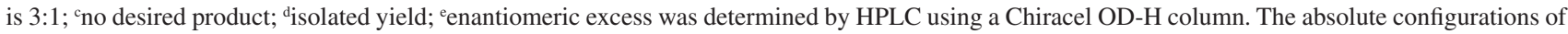
all products were determined by comparing the retention time from HPLC data on the products with those in the literature data. ${ }^{19-21}$

chiral calix[4]arenes containing quinolin-2-yl-methanol moiety. ${ }^{2}$ According to the proposed mechanistic mode by Deng and co-workers, ${ }^{15}$ we believe that the aldehyde is activated by the hydroxyl group through hydrogen bonding, while the nitromethane is activated by the tertiary amino group in $\mathbf{5 a}$ or $\mathbf{5 b}$ catalytic process (Scheme 2). The more beneficial effect of the protic solvent, especially the mixed solvent $\mathrm{EtOH} / \mathrm{H}_{2} \mathrm{O}$, may be plausibly explained that they can effectively stabilize nitromethide anion and nitro aldol product through hydrogen bonding interaction.

Lowering reaction temperature has frequently been reported to enhance the enantioselectivity of Henry

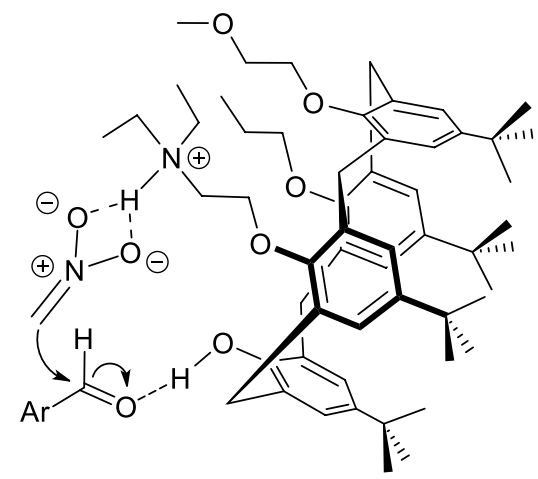

Scheme 2. Proposed mode of action of catalyst $\mathbf{5 a}$. reaction..$^{9-11}$ With the optimized solvents in hand, the effect of temperature on enantioselectivity in the Henry reaction is also studied in a mixed protic solvent $\left(\mathrm{EtOH} / \mathrm{H}_{2} \mathrm{O}, 3: 1, \mathrm{v} / \mathrm{v}\right)$. The results from the different temperatures (Table 1) showed the enantioselectivity can not be further improved by lowering the temperature at the accompanying expense of increasing the reaction time. At $-10{ }^{\circ} \mathrm{C}$, the enantioselectivities became poorer $(1.7 \%$ ee using $\mathbf{5 a}$ and $1.2 \%$ ee using $\mathbf{5 b}$ ) and the reaction times were increased to $24 \mathrm{~h}$ although the yields were remarkably improved (Table 1, entries 7 and 8 versus entries 11 and 12). At $-25^{\circ} \mathrm{C}$, the enantioselectivities can not be observed and the reaction times were further increased to $72 \mathrm{~h}$ although the yield changes were not obvious (Table 1, entries 7 and 8 versus entries 13 and 14).

Under the optimal reaction conditions ( $5 \mathrm{~mol} \%$ catalyst, room temperature and a mixed protic solvent $\mathrm{EtOH} / \mathrm{H}_{2} \mathrm{O}$ ), we then explored a variety of aromatic aldehydes using the catalyst 5a. Compared to 4-nitrobenzaldehyde, the aromatic aldehydes bearing electron-withdrawing groups (4-CN, 4-F, 2- $\mathrm{NO}_{2}, 3-\mathrm{NO}_{2}$ and 3-Cl) produced comparable yields and lower enantiomeric excesses (Table 2, entries 1-5). However, the catalytic results from the aromatic aldehydes bearing electron-donating groups $(4-\mathrm{MeO}$, 
Table 2. 5a-promoted Henry reaction of nitromethane with different aromatic aldehydes

\begin{tabular}{|c|c|c|c|c|c|}
\hline entry ${ }^{a}$ & $\mathrm{R}$ & time $/ \mathrm{h}$ & Configuration & Yield $^{\mathrm{c}} / \%$ & $\mathrm{ee}^{\mathrm{d} / \%}$ \\
\hline 1 & $4-\mathrm{CN}$ & 36 & $S$ & 98 & 2.1 \\
\hline 2 & 4-F & 36 & $S$ & 85 & 0.2 \\
\hline 3 & $2-\mathrm{NO}_{2}$ & 12 & $S$ & 87 & 1.3 \\
\hline 4 & $3-\mathrm{NO}_{2}$ & 16 & $S$ & 83 & 1.2 \\
\hline 5 & $3-\mathrm{Cl}$ & 24 & $S$ & 80 & 2.7 \\
\hline 6 & $4-\mathrm{MeO}$ & 48 & $S$ & 25 & 1.0 \\
\hline 7 & $4-\mathrm{N}\left(\mathrm{CH}_{3}\right)_{2}^{\mathrm{b}}$ & 48 & - & - & - \\
\hline 8 & $2-\mathrm{CH}_{3}$ & 48 & $S$ & 68 & 0.5 \\
\hline
\end{tabular}

aThe reaction was performed with $0.6 \mathrm{mmol}$ aromatic aldehyde and $6 \mathrm{mmol}$ nitromethane in $4 \mathrm{~mL}$ solvent $\left(\right.$ EtOH: $\mathrm{H}_{2} \mathrm{O}, 3: 1$, v/v); ${ }^{b}$ no reaction; ${ }^{\mathrm{i}}$ isolated yield; denantiomeric excess was determined by HPLC using a Chiracel OD-H column. The absolute configurations of all products were determined by comparing the retention time from HPLC data on the products with those in the literature data. ${ }^{19-21}$

4- $\mathrm{N}\left(\mathrm{CH}_{3}\right)_{2}$ and 2- $\left.\mathrm{CH}_{3}\right)$ showed comparatively poorer. 4-Methoxybenzaldehyde and 2-methylbenzaldehyde only afforded $25 \%$ yield and $1.2 \%$ ee, and $68 \%$ yield and $0.5 \%$ ee in $48 \mathrm{~h}$ (Table 2, entries 6 and 8). Although a large quantity of the two substrates remained unreacted, their reactions were clean, and we did not observe dehydration to the corresponding nitroalkenes. The reaction with 4-dimethylaminobenzaldehyde can not take place in the tested condition. These catalytic results clearly showed that catalyst $\mathbf{5 a}$ and the optimized reaction conditions can be applied in a wide scope of aromatic aldehydes, and electron-withdrawing groups on aromatic ring are more beneficial to Henry reaction than electron-donating ones.

In $\mathbf{5 a}$ and $\mathbf{5 b}$, the ethylene links and methyl groups of their catalytic amino groups are free to rotate and the anchoring phenoxyl group can flap into and away from the calix cavity. Therefore, it can be conceived that their catalytic amino groups are also free to rotate. The nonsatisfactory solvent-dependent enantioselectivity may mainly be attributed to the high flexibility of their catalytic amino groups. Reducing the multiple factors of the high flexibility of the catalytic amino groups will be a huge challenge in the structural modification of inherently chiral calix[4]arenes substituted at the lower rim. So many heavy efforts have been made to structurally modify inherently chiral calix[4]arenes substituted at the meta position and the upper rim in Chen and Huang group ${ }^{1,2}$ and Shimizu group..$^{3-7}$ However, the enantioselectivities in their asymmetric catalysis processes are always poor. Therefore, the asymmetric catalysis prospects of inherently chiral calix[4]arenes seem somewhat gloomy.

\section{Conclusions}

In conclusion, starting from a pair of reported inherently chiral calix[4]arene diastereomers, a pair of $\mathrm{N}, \mathrm{O}$-type enantiomers based on inherently chiral calix[4] arenes substituted at the lower rim were synthesized. Their organocatalytical behaviors on Henry reaction between aromatic aldehydes and nitromethane were studied in different solvent under different temperature. The aromatic aldehydes bearing electron-withdrawing groups, a mixed protic solvent and room temperature are beneficial for the organocatalytic reaction. The high flexibility of their catalytic amino groups may mainly result in the nonsatisfactory solvent-dependent enantioselectivity.

\section{Supplementary Information}

Supplementary information (NMR spectra of new compounds and HPLC spectra) is available free of charge at http://jbcs.sbq.org.br as PDF file.

\section{Acknowledgments}

We thank the National Natural Science Foundation of China (No. 21272173) for financial support.

\section{References}

1. Xu, Z.-X.; Li, G.-K.; Chen, C.-F.; Huang, Z.-T.; Tetrahedron 2008, 64, 8668 . 
2. Miao, R.; Xu, Z.-X.; Huang, Z.-T.; Chen, C.-F.; Sci. China Chem. 2009, 52, 505.

3. Shirakawa, S.; Moriyama, A.; Shimizu, S.; Org. Lett. 2007, 9 , 3117.

4. Shirakawa, S.; Moriyama, A.; Shimizu, S.; Eur. J. Org. Chem. 2008, 5957.

5. Shirakawa, S.; Shimizu, S.; Eur. J. Org. Chem. 2009, 1916.

6. Shirakawa, S.; Kimura, T.; Murata, S.; Shimizu, S.; J. Org. Chem. 2009, 74, 1288.

7. Shirakawa, S.; Shimizu, S.; New J. Chem. 2010, 34, 1217.

8. Li, S. Y.; Xu, Y. W.; Liu, J. M.; Su, C. Y.; Int. J. Mol. Sci. 2011, $12,429$.

9. Boruwa, J.; Gogoi, N.; Saikia, P. P.; Barua, N. C.; Tetrahedron: Asymmetry 2006, 17, 3315.

10. Palomo, C.; Oiarbide, M.; Laso, A.; Eur. J. Org. Chem. 2007, 2561.

11. Alvarez-Casao, Y.; Marques-Lopez, E.; Herrera, R. P.; Symmetry 2011, 3, 220.

12. Christensen, C.; Juhl, K.; Jørgensen, K. A.; Chem. Commun. 2001, 2222.
13. Blay, G.; Hernandez-Olmos, V.; Pedro, J. R.; Synlett 2011, 1195.

14. Milner, S. E.; Moody, T. S.; Maguire, A. R.; Eur. J. Org. Chem. 2012, 3059.

15. Li, H.; Wang, B.; Deng, L.; J. Am. Chem. Soc. 2006, 128, 732.

16. Marcelli, M.; van der Haas, R. N. S.; van Maarseveen, J. H.; Hiemstra, H.; Synlett 2005, 2817.

17. Yesypenko, O. A.; Boyko, V. I.; Shishkinb, O. V.; Shishkina, S. V.; Pirozhenko, V. V.; Kalchenko, V. I.; Russ. J. Org. Chem. 2012, 48, 284.

18. Jaime, C.; de Mendoza, J.; Prados, P.; Nieto, P. M.; Sanchez, C.; J. Org. Chem. 1991, 56, 3372.

19. Evans, D. A.; Seidel, D.; Rueping, M.; Lam, H. W.; Shaw, J. T.; Downey, C. W.; J. Am. Chem. Soc. 2003, 125, 12692.

20. Spangler, K. Y.; Wolf, C.; Org. Lett. 2009, 11, 4724.

21. Bulut, A.; Aslan, A.; Dogan, Ö.; J. Org. Chem. 2008, 73, 7373.

Submitted: September 30, 2016

Published online: November 24, 2016 